\title{
Monte Carlo study on ferromagnetic phase transition of two-dimensional Ising model
}

\author{
Zhao Hui Min ${ }^{1, a, *}$ \\ ${ }^{1}$ Bohai University, Jinzhou 121013, China \\ ahmzhao2008@163.com
}

\begin{abstract}
Keywords: Monte Carlo simulation, Phase transition, Ising model, Binder ratio
Abstract. We have investigated the phase transition of two-dimensional Ising model by Monte Carlo method. Our results reveal that a finite-temperature phase transition occurs in the Ising model. The calculation result agrees well with the theoretical one. The specific heat and the susceptibility peak at the critical temperature while they descend at both high and low temperature. The spontaneous magnetization approaches to unit when the temperature is below the critical temperature.
\end{abstract}

\section{Introduction}

In real world, matter has different states. For instance, gas, liquid, solid, magnetic ordering, superconducting and superfluid states can exist in different materials. When the matter transits from one state to another state, phase transition occurs in the system. According to the derivative of free energy, the phase transition can be classified to first order and second order [1,2]. Essentially, phase transition originates from the destroyed symmetry. When the high symmetry is destroyed or lowed, phase transition occurs. For example, when the time-reversal symmetry is destroyed in the system, the magnetic phase transition occurs in it. Therefore, study on the spontaneous magnetization of magnetic interacting system has an important effect on the theory of phase transition. According to the theory of phase transition, proposed first by Landau, free energy can be expressed as the even power series of order parameter. When the phase transition occurs, the order parameter arises simultaneously. For instance, when magnetic phase transition occurs, the magnetic order, i.e. magnetization, arises. When the superconducting current occurs, the Cooper pairs of electron arise. When helium is cooled below $4.2 \mathrm{~K}$, the component of superfluid appears. Monte Carlo method can be used to simulate the magnetic transition of interacting magnetic system. Monte Carlo method is a powerful method based on random number. It was used to simulate the article transportation, magnetic phase transition[3], integration, engineering and economic problems. Monte Carlo method can be used to simulate the phase transition as well as the critical behaviors and finite-size effects. In this article, we use Monte Carlo method to study the magnetic phase transition of two-dimensional (2D) Ising model. The critical temperature as well as the critical behaviors is also studied.

\section{Calculation method}

We use Monte Carlo method to investigate the magnetic phase transition of 2D Ising model. The lattice size of $L \times L$ is used in the simulation and $L$ takes 20,30, 40 and 50. Spin on each site takes the values of +1 or -1 . The value of +1 corresponds to up spin state and the value of -1 corresponds to down spin state. The Hamiltonian of the system can be written as

$$
H=-J \sum_{i \neq j} \sigma_{i} \cdot \sigma_{j}
$$

The exchange parameter $\mathrm{J}$ takes 1.0 and $\sigma_{i}$ is the spin of unit on the ith site. Therefore, the Hamiltonian describes the ferromagnetic interacting spin system. The above magnetic interacting system can be modeled as canonical ensemble. In canonical ensemble, the ith spin occupies the state with energy $E_{i}$ according to the probability

$$
\rho_{i}=e^{-E_{i} / k_{B} T} / \sum_{i} e^{-E_{i} / k_{B} T}
$$

We use the Metropolis sampling method to describe the spin transiting from one state to other state under the thermal fluctuation. According to the Metropolis sampling method, spin flips as follows: (i) 
energy difference of $\Delta E$ is calculated between the states with energy of $E_{i}$ and $E_{j}$; (ii) calculate the relative transiting probability $p=e^{-\Delta E / k_{B} T}$; (iii) random number $r$ is generated. If $r<p$, spin flips from the state with energy of $E_{i}$ to the state with energy of $E_{j}$, that is, the transition of $E_{i} \rightarrow E_{j}$ is permitted. If $r>p$, the reversal proposal is rejected.

To study the magnetic phase transition of 2D Ising model, we calculated the magnetization, specific heat, magnetic susceptibility and Binder ratio. These expressions can be written as $[4,5]$

$$
\begin{aligned}
& C_{v}=(E-<E>)^{2} /\left(k_{B} T^{2}\right) \\
& \chi=(\sigma-<\sigma>)^{2} /\left(k_{B} T\right) \\
& B_{r}=1.0-\frac{<\sigma^{4}>}{3<\sigma^{2}>^{2}}
\end{aligned}
$$

In the above equations, the angle brackets $<\ldots \ldots>>$ represent the thermodynamic average of physical quantities. Therefore, $\langle E\rangle$ represents average energy, $\langle\sigma\rangle$ is the average magnetization. In the simulation, $5 \times 10^{5}$ Monte Carlo steps are used to sweep the Ising system and $5 \times 10^{5}$ Monte Carlo steps are used for the sampling of quantities. The temperature takes the unit of energy and the temperature region in simulation ranges from 1.8 to 2.8 , and the temperature interval takes 0.01 .

\section{Calculation results}

For 2D Ising system, we simulate that the system was cooled from high temperature to low temperature without external magnetic field. Our results reveal that the system undergoes ferromagnetic transition near the critical temperature. The magnetization versus the temperature is shown in Fig. 1.

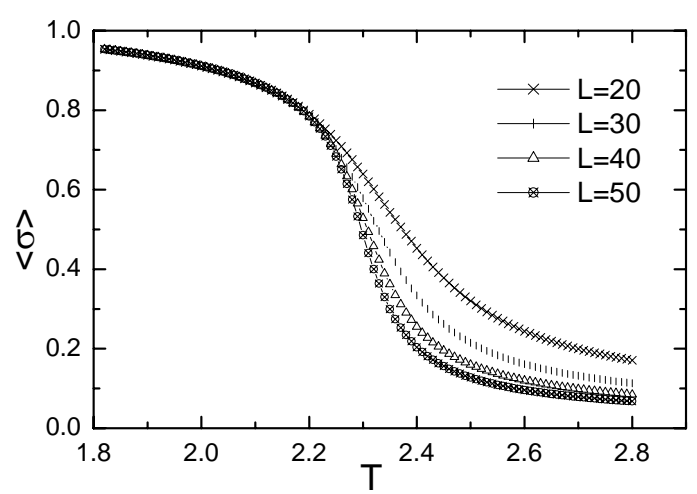

Fig.1: Spontaneous magnetization versus temperature for Ising system with different size.

As shown in Fig. 1, the magnetization becomes larger and larger as the temperature decreases. When the temperature is below 2.2, the magnetization reaches to 0.8 , that is, the system reaches to the magnetic ordering state. Moreover, as the size of lattice increases, the magnetization near the critical temperature increases more abruptly. Near the critical temperature, the order parameter fluctuates greatly and the magnetization increases largely as the temperature decreases. As the temperature decreases below the critical temperature, the magnetization approaches to 1.0. At low temperature, thermal fluctuation has less effect on the spin flipping and all the spins are aligned to one direction under the ferromagnetic interaction.

Figure 2 shows the magnetic specific heat versus temperature for different lattices. 


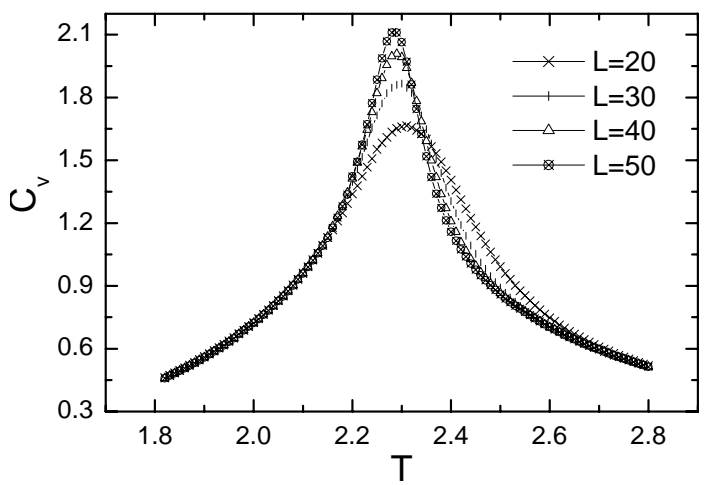

Fig.2: Specific heat versus temperature for different Ising model.

As shown in Fig. 2, the specific heat peak emerges near the temperature. In contrast, the specific heat descends at both low and high temperatures. Essentially, specific heat is contributed by energy fluctuation. Near the critical temperature, the size of order parameter nearly approaches to the size of system and the size destroyed by thermal fluctuation is also proportional to it. Therefore, at the critical temperature, the system energy fluctuates greatly and the peak of specific heat emerges. As can be seen from Fig. 2, the specific heat peaks abruptly with the size increasing.

The susceptibility versus temperature is shown in Fig. 3 for Ising system with different size.

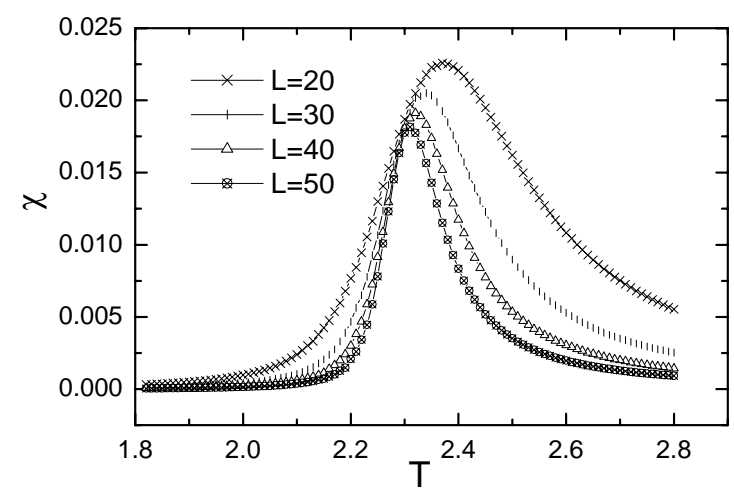

Fig. 3: Magnetic susceptibility versus temperature for Ising system with different size.

Similar to the behavior of specific heat, the susceptibility peaks at the critical temperature. Near the temperature, the peak shifts to low temperature and becomes sharp as the size of system increases. Due to the great spin fluctuation near the critical temperature, the magnetization fluctuation also becomes large. Therefore, the susceptibility peaks at the critical temperature.

Figure 4 shows the Binder ratio versus the temperature for Ising system with different size. Due to the sensitivity of Binder ratio, it changes significantly as the temperature and the size of system varies. Therefore, the Binder ratio can be used to determine the critical temperature accurately.

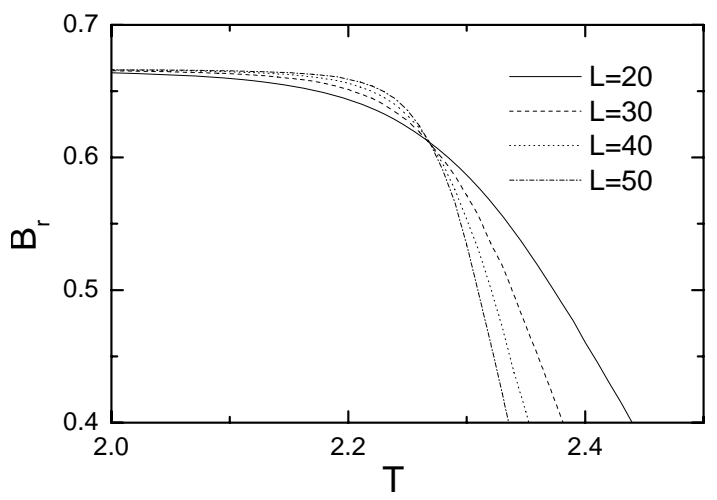

Fig. 4: Binder ratio versus temperature for Ising system with different size. 
It is noticed that the Binder ratio approaches to zero at high temperature while it approaches to 2/3 at low temperature. The curves of Binder ratio cross at 2.265, which means that the critical temperature $k_{B} T_{c}=2.265 \mathrm{~J}$. This agrees well with the theoretical result of $k_{B} T_{c}=2.26 \mathrm{~J}$ obtained by Onsager [1,2]. Our results reveal that Monte Carlo simulation can well reproduce the magnetic phase transition of canonical magnetic interacting system.

\section{Conclusions}

We use Monte Carlo method to simulate the magnetic phase transition of 2D Ising model. Our results reveal that the quantities of magnetization, specific heat and susceptibility peak at the critical temperature. Our results demonstrate that the finite-temperature phase transition does occur in the 2D Ising system. By calculating the Binder ratio of the Ising system, the ratio curves with different size cross at $k_{B} T_{c}=2.265 \mathrm{~J}$. This agrees well with the theoretical value of Onsager. This demonstrates that Monte Carlo method is a powerful method to simulate the phase transition of canonical magnetic system.

\section{Acknowledgment}

This work is supported by Social Science Foundation of Liaoning Province (No. L15DWW005) and Bohai University Teaching Reform Project (No.BDJG15QNB002).

\section{References}

[1] K. Huang, Statistical Mechanics, second ed., New York: John Wiley and Sons, 1987.

[2] L.E. Reichl, Modern Course in Statistical Physics, Austin: University of Texas,1980.

[3] K.C. Zhang, P.-Y. Song,Spin glass dynamics in RKKY interacting disordered magnetic system. Chin Phys B 19(2010): 097105.

[4] D.P. Laudau, K. Binder, A Guide to Monte Carlo Simulations in Statistical Physics, Cambridge University Press, 2008, pp.78-79

[5] K. Binder and A. P. Young, Spin glasses: Experimental facts, theoretical concepts, and open questions, Rev. Mod. Phys. 58(1986): 801. 